\title{
Enrichment of Deuterium Oxide at Hydrophilic Interfaces in Aqueous Solutions
}

\author{
Johan J.R. Stålgren ${ }^{1}$, Katrin Boschkova ${ }^{2}$, Jan-Christer Ericsson ${ }^{3}$, Curtis W. Frank ${ }^{1}$, \\ Wolfgang Knoll $^{4}$, Sushil Satija ${ }^{5}$, Michael F. Toney ${ }^{6}$ \\ ${ }^{1}$ Department of Chemical Engineering, Stanford University, Stanford, CA 94305, USA \\ ${ }^{2}$ Institute for Surface Chemistry, Box 5607, SE-114 86 Stockholm, Sweden \\ ${ }^{3}$ Department of Chemistry, Surface Chemistry, Royal Institute of Technology, Box 5607, \\ SE-100 44 Stockholm, Sweden \\ ${ }^{4}$ Max Planck Institute for Polymer Research, Ackermannweg 10, 55128 Mainz, Germany \\ ${ }^{5}$ NIST Center for Neutron Scattering, National Institute of Standards \& Technology \\ Gaithersburg MD 20899, USA \\ ${ }^{6}$ Stanford Synchrotron Radiation Laboratory, Stanford Linear Accelerator Center, Menlo \\ Park, CA 94025, USA
}

Supporting Information

The neutron reflectivity experiment used a quartz block substrate ( $0.4 \mathrm{~nm}$ roughness), while the QCM used a sputtered film of $\mathrm{SiO}_{2}$ (1.2 $\mathrm{nm}$ roughness). Since our experiments probe the interfacial water layer over lengths $1-10 \mathrm{~nm}$ perpendicular to the interface (i.e., larger than the roughness), we do not expect any differences in this layer due to the different roughness of these surfaces. The quartz substrate was initially cleaned as follows: immersion in a mixture of $25 \% \mathrm{NH}_{4}, 30 \% \mathrm{H}_{2} \mathrm{O}_{2}$, and $\mathrm{H}_{2} \mathrm{O}$ at $80^{\circ} \mathrm{C}$ for 10 minutes, rinse with water, immersion in $25 \% \mathrm{HCl}, 30 \% \mathrm{H}_{2} \mathrm{O}_{2}$ and $\mathrm{H}_{2} \mathrm{O}$ at $80^{\circ} \mathrm{C}$ for 10 minutes, final rinse with water and dry in nitrogen. Between neutron reflectivity experiments, we used a simpler cleaning procedure: rinsing with ethanol, drying with flowing nitrogen, and 20 minutes in a UV-chamber followed again with flowing nitrogen. This simpler procedure was used to ensure a reproducible surface between the experiments, since the initial cleaning procedure with acid and base solutions is quite aggressive. After every experiment, an ocular inspection was performed to assess the surface wetting properties. Since the surface showed no dewetting, we felt confident in applying the simple cleaning with ethanol. The exposure of the surface to ambient after each simple cleaning was less than one minute before it was placed in our solution, thus guaranteeing a minimal exposure to airborne contaminants from outside the laminar flow 
hood. Water used was of Millipore Milli-Q Plus 185 ultra-pure quality, deuterium oxide 99.8 atom \% D, from Sigma-Aldrich.

Specular neutron reflectivity data were obtained at the NG-7 reflectometer at the Center for Neutron Research at the National Institute of Standards and Technology using a neutron wavelength of $0.476 \mathrm{~nm}$. The quartz sample was held horizontally and the scattering plane was vertical. The neutrons pass through the quartz sample and then reflect at the quartz-solution interface. ${ }^{1-4}$ All measurements were at $21^{\circ} \pm 0.5 \mathrm{C}$. The diffuse background was carefully determined through both offset scans and though rocking scans. ${ }^{5}$ This diffuse scattering was subtracted from the intensity data and these data were then normalized by the incident beam intensity to yield the specular reflectivity. Our pure $\mathrm{D}_{2} \mathrm{O}$ solution absorbed ambient $\mathrm{H}_{2} \mathrm{O}$ during the solution preparation process for the neutron measurements resulting in a $98 \% \mathrm{D}_{2} \mathrm{O}$ solution. However, for simplicity, we refer to this as pure $\mathrm{D}_{2} \mathrm{O}$. Specular neutron reflectivity measures the neutron scattering length density laterally averaged over $20 \mu \mathrm{m}$ with an effective depth resolution of several $\mathrm{nm}^{6}$.

The neutron reflectivity are analyzed using the well-established models based on the Parratt formalism ${ }^{1,6,7}$. The model includes Gaussian surface roughness at the quartz/solution interface and an interfacial layer with a uniform scattering length density next to the interface and an exponential tail into the bulk solution (see Fig. 2). A summary of the best fit parameters appears in Table S1.We have used an exponential to describe the decay into the bulk solution because this is physically plausible. We note, however, that we cannot rule out other profiles since the reflectivity data do not extent to very high $\mathrm{Q}$. There are no oscillations apparent in the data, as one might expect for a layered system, since the 'interface' between the $\mathrm{D}_{2} \mathrm{O}$-rich layer and the bulk solution is diffuse. This diffuseness and the small Q range limits our ability to accurately model the extent of the enriched layer. Thus, the error bars are these quantities are rather large (see Table S1). We fixed the exponential lengths for the data at $5 \%$ and $10 \% \mathrm{D}_{2} \mathrm{O}$ to values that are larger than for the fits for the higher concentrations, since if we allowed these to vary they would diverge. In modeling the neutron reflectivity data, there is a coupling 
between some of the fitting parameters, specifically the interfacial layer thickness, the exponential length, and the interfacial layer SLD. Hence, the individual error bars in these parameters are large (see Table S1). However, the surface excess calculated in Fig. $4 \mathrm{~b}$ is more robust in the fitting and thus has smaller error bars.

Figure $\mathrm{S} 1$ shows neutron reflectivity for $5 \%, 15 \%$ and $30 \% \mathrm{D}_{2} \mathrm{O}$ solutions along with the best fits (solid lines) and 'best-fit' models assuming no enriched- $\mathrm{D}_{2} \mathrm{O}$ interfacial layer (dashed lines) but with an interfacial layer of adventiously adsorbed hydrocarbons (contamination). Such adsorbed layers are typically found for $\mathrm{SiO}_{2} / \mathrm{Si}$ in air and are $0.5-1$ $\mathrm{nm}$ thick $^{8}$. This contamination layer is modeled with a $0.5 \mathrm{~nm}$ layer with $\mathrm{SLD}=-3.56 \times 10^{-7}$ $\AA^{-2}$ (appropriate for $\mathrm{CH}_{2}$ ) but we have tried other SLDs for contamination layers (0.5-1.0 nm with SLDs of $-4 \times 10^{-7} \AA^{-2}$ to $5 \times 10^{-7} \AA^{-2}$, which is appropriate for $\mathrm{CH}$ ). As is apparent in Fig. S1, these fail to fit the data. We also note that to explain our data any impurity layer would have to have an SLD that depends on the bulk D2O/H2O concentration (see Fig. 3), which seems unlikely. Furthermore, such contamination cannot explain our QCM data.

Quartz crystals (Q-Sense AB, Västra Frölunda, Sweden) with a gold coating, were used for the QCM measurements. These had a fundamental resonant frequency of $4.95 \mathrm{MHz}$. A $100 \mathrm{~nm}$ film of $\mathrm{SiO}_{2}$ was sputtered onto the gold surface and had an rms surface roughness of $1.2 \pm 0.2 \mathrm{~nm}$ (measured with white-light interferometric profilometry). QCM measurements were in a temperature controlled $\left(21^{\circ} \pm 0.05 \mathrm{C}\right)$ closed chamber. We used the QCM to simultaneously measure the changes in frequency and dissipation at the first four harmonics, since this is a proven way to verify the acquired data and hence improved the accuracy of each experiment.

\section{References}

(1) Fragneto, G.; Lu, J. R.; McDermott, D. C.; Thomas, R. K.; Rennie, A. R.; Gallagher, P. D.; Satija, S. K. Langmuir 1996, 12, 477-486.

(2) Schwendel, D.; Hayashi, T.; Dahint, R.; Pertsin, A.; Grunze, M.; Steitz, R.; Schreiber, F. Langmuir 2003, 19, 2284-2293.

(3) Forciniti, D.; Hamilton, W. A. J. Coll. Inter. Sci. 2005, 285, 458-468. 
(4) Burgess, I.; Li, M.; Horswell, S. L.; Szymanski, G.; Lipkowski, J.; Majewski, J.; Satija, S. Biophys. J. 2004, 86, 1763-1776.

(5) Toney, M. F.; Thompson, C. J. Chem. Phys. 1990, 92, 3781-3793.

(6) Dura, J. A.; Richter, C. A.; Majkrzak, C. F.; Nguyen, N. V. Appl. Phys. Lett. 1998, 73, 2131-2133.

(7) Lu, J. R.; Thomas, R. K. Journal of the Chemical Society-Faraday Transactions 1998, 94, 995-1018.

(8) Toney, M. F.; Mate, C. M.; Pocker, D. Ieee Transactions on Magnetics 1998, 34, 1774-1776. 


\begin{tabular}{|l|l|l|l|l|}
\hline $\begin{array}{l}\mathrm{D}_{2} \mathrm{O} \\
\text { concentration }\end{array}$ & $\begin{array}{l}\text { Bulk solution } \\
\mathrm{SLD}\left(10^{-6} \AA^{-2}\right)\end{array}$ & $\begin{array}{l}\text { Interfacial layer } \\
\text { SLD }\left(10^{-6} \AA^{-2}\right)\end{array}$ & $\begin{array}{l}\text { Interfacial layer } \\
\text { thickness }(\mathrm{nm})\end{array}$ & $\begin{array}{l}\text { Exponential } \\
\text { decay }(\mathrm{nm})\end{array}$ \\
\hline 0.0 & -0.56 & $\mathrm{n} / \mathrm{a}$ & $\mathrm{n} / \mathrm{a}$ & \\
\hline 0.05 & -0.18 & $0.26(0.1)$ & 7.1 & $5.0^{*}$ \\
\hline 0.10 & 0.21 & $0.90(0.15)$ & 5.1 & $6.0^{*}$ \\
\hline 0.15 & 0.58 & $1.9(0.3)$ & 2.5 & 2.5 \\
\hline 0.20 & 0.95 & $2.2(0.3)$ & $3.0^{*}$ & 2.7 \\
\hline 0.30 & 1.68 & $2.6(0.3)$ & $3.0^{*}$ & 3.0 \\
\hline 0.50 & 3.07 & $3.9(0.3)$ & $2.5^{*}$ & 1.7 \\
\hline 0.70 & 4.51 & $5.1(0.3)$ & 2.0 & 2.6 \\
\hline $1.0(0.98)$ & 6.24 & $\mathrm{n} / \mathrm{a}$ & $\mathrm{n} / \mathrm{a}$ & $\mathrm{n} / \mathrm{a}$ \\
\hline
\end{tabular}

Table S1. Best fit parameters from neutron reflectivity modeling. SLD is the neutron scattering length density. A* indicates the parameter was fixed because the fit was reasonably insensitive to this and the parameter would diverge if not fixed. These values are fixed close to those found for nearby $\mathrm{D}_{2} \mathrm{O}$ concentrations. For the data at $5 \%$ and $10 \%$ $\mathrm{D}_{2} \mathrm{O}$ concentration, the exponential decay lengths are set to values that are larger than for the fits for the higher concentrations, since if we allowed these to vary they would diverge to unphysically large values $(>100 \mathrm{~s} \mathrm{~nm})$. The estimated error bars on the interfacial layer thickness is about $2-3 \mathrm{~nm}$ and for the exponential decay is $3-4 \mathrm{~nm}$. The estimated error bars for the Interfacial layer SLDs are given in the (). 


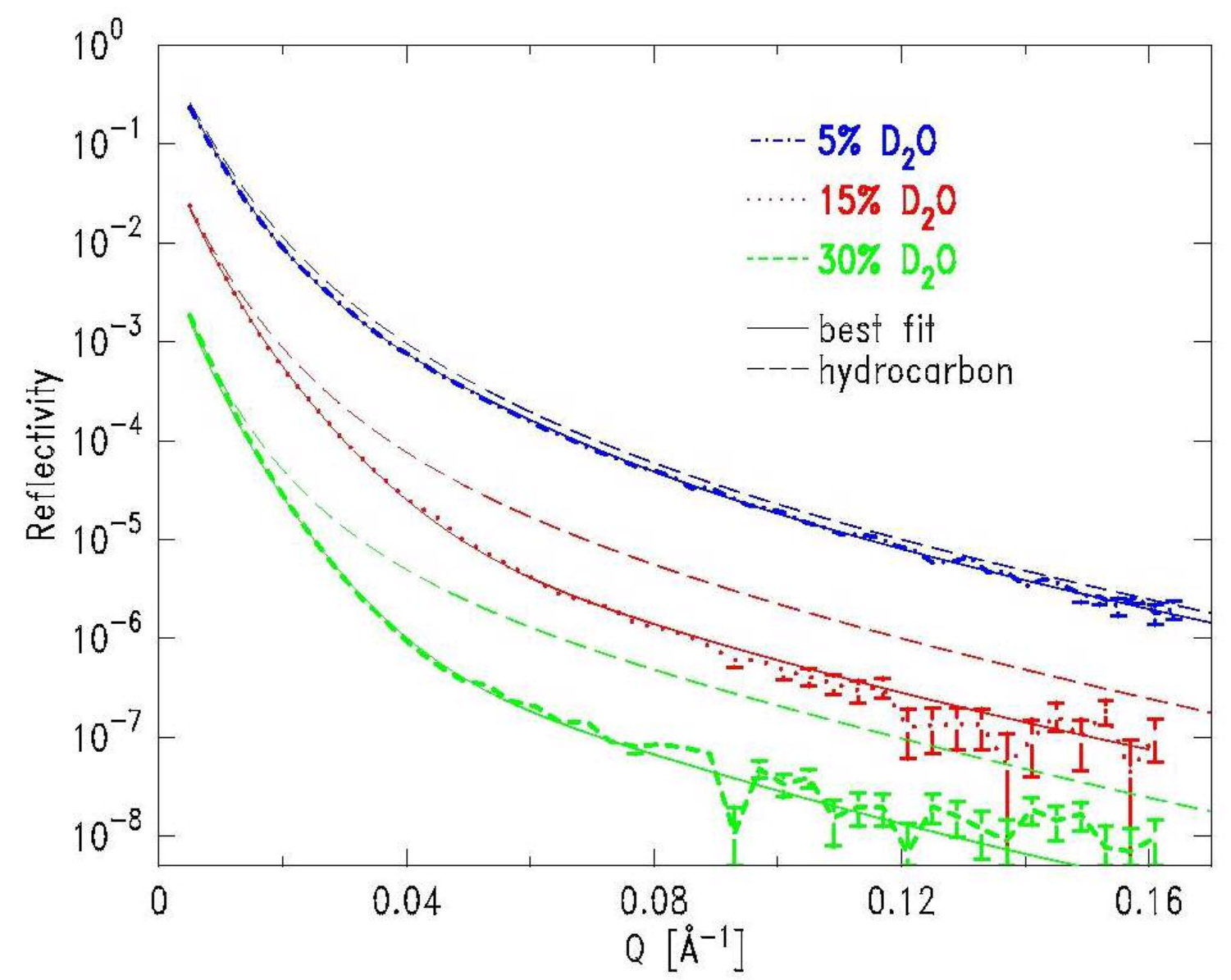

Figure S1. Measured (thick lines) and fitted neutron reflectivity for the quartz- $\mathrm{H}_{2} \mathrm{O} / \mathrm{D}_{2} \mathrm{O}$ mixtures $(5 \%, 15 \%$ and $30 \%)$. The best fits with the enriched- $\mathrm{D}_{2} \mathrm{O}$ layer are shown by the thin solid lines, while the fits with a $0.5 \mathrm{~nm}$ hydrocarbon interfacial layer are the thin, dashed lines. Data are offset for clarity. 